\title{
Four Families of Folate-independent Methionine Synthases
}

\author{
Morgan N. Price, Adam M. Deutschbauer, and Adam P. Arkin \\ Lawrence Berkeley National Lab
}

\section{Abstract}

Although most organisms synthesize methionine from homocysteine and methyl folates, some have "core" methionine synthases that lack folate-binding domains and use other methyl donors. In vitro, the characterized core synthases use methylcobalamin as a methyl donor, but in vivo, they probably rely on corrinoid (vitamin B12-binding) proteins. We identified four families of core methionine synthases that are distantly related to each other (under 30\% pairwise amino acid identity). From the characterized enzymes, we identified the families MesA, which is found in methanogens, and MesB, which is found in anaerobic bacteria and archaea with the Wood-Ljungdahl pathway. A third uncharacterized family, MesC, is found in anaerobic archaea that have the Wood-Ljungdahl pathway and lack known forms of methionine synthase. We predict that most members of the MesB and MesC families accept methyl groups from the iron-sulfur corrinoid protein of that pathway. The fourth family, MesD, is found only in aerobic bacteria. Using transposon mutants and complementation, we show that MesD does not require 5-methyltetrahydrofolate or cobalamin. Instead, MesD requires an uncharacterized protein family (DUF1852) and oxygen for activity.

\section{Introduction}

Methionine is required for protein synthesis and is also a precursor to S-adenosylmethionine, which is the methyl donor for most methyltransferases and is required for polyamine biosynthesis. Methionine is synthesized from aspartate by reduction and sulfhydrylation to homocysteine, and then the transfer of a methyl group to homocysteine to give methionine (Figure 1). There are two well-studied forms of methionine synthase, both of which obtain the methyl group from 5-methyltetrahydrofolates (5-methyl-THF). MetH requires cobalamin (vitamin B12) or other cobamides as a cofactor, while MetE does not require any organic cofactor. For the same mass of protein, MetH is about 40 times more active than MetE (González et al. 1996; Goulding et al. 1997). Escherichia coli, which cannot synthesize cobamides, has both enzymes. There are also methyltransferases that convert homocysteine to methionine by using methylated nutrients such as glycine betaine or S-methylmethionine (Caspi et al. 2010). Here we will focus on the synthesis of methionine without special nutrient requirements. 


\section{A. Standard pathway for methionine synthesis}

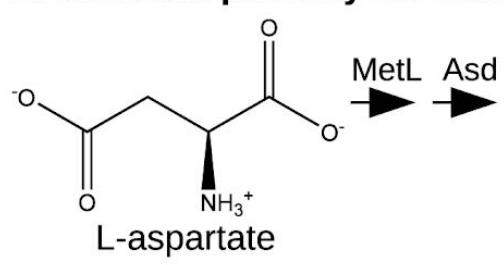<smiles>N[C@@H](CC=O)C(=O)O</smiles>

L-aspartate 4-semialdehyde

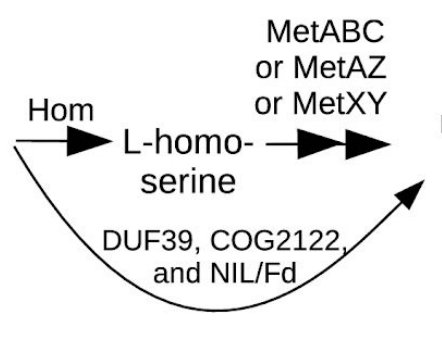

MetABC

or MetAZ<smiles>[NH3+][C@@H](CCS)C(=O)O</smiles>

L-homocysteine

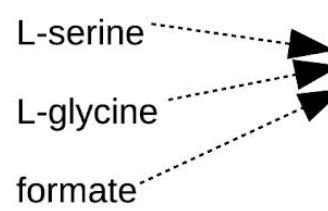

B. Core methionine synthases
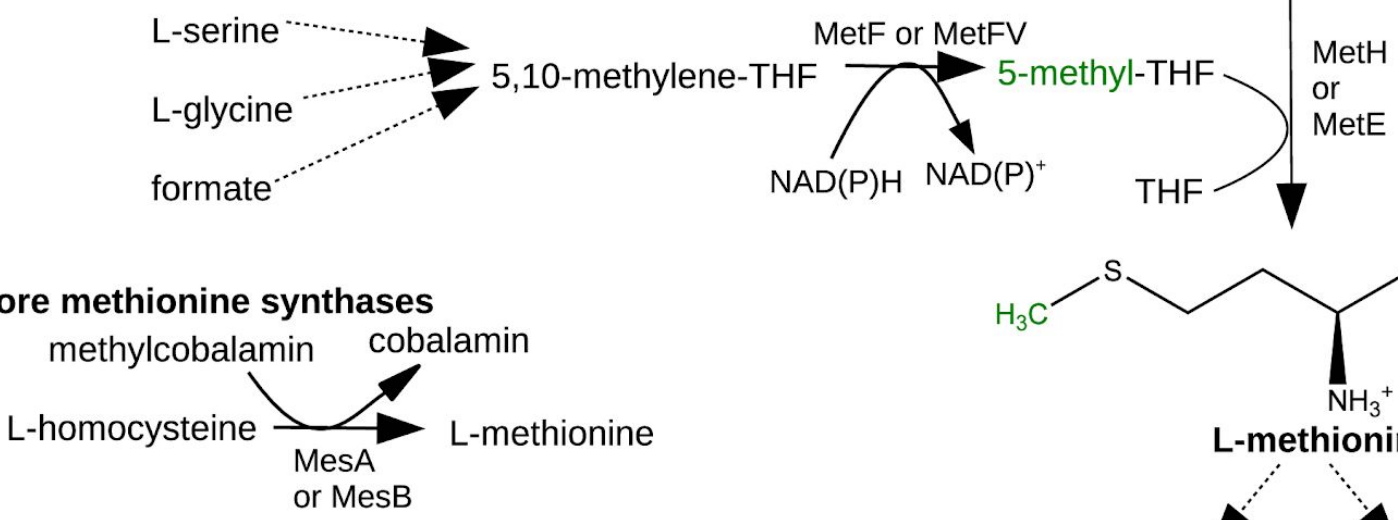
5,10-methylene-THF<smiles>C=C([O-])[C@@H]([NH3+])CCSC</smiles>

L-methionine

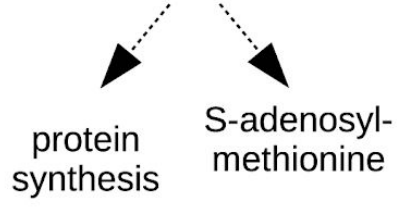

C. Structure of methylcobalamin

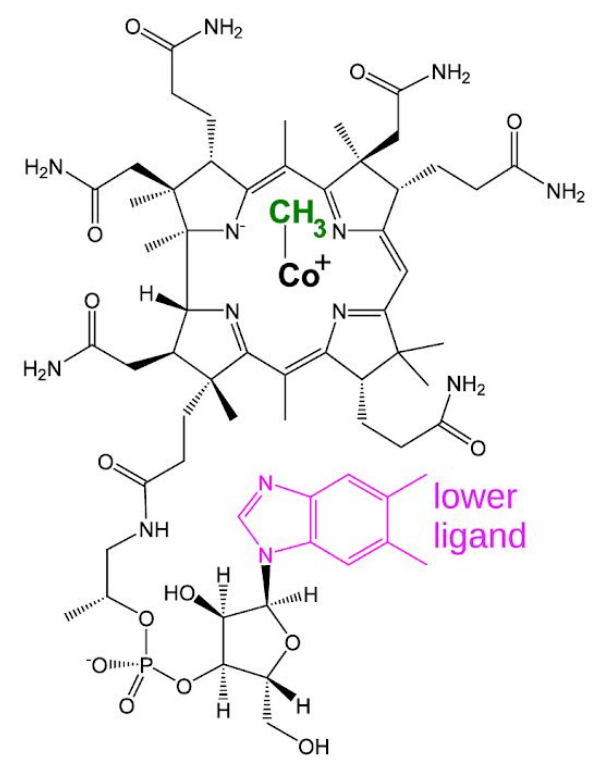

D. Structure of 5-methyl-tetrahydrofolate

Figure 1: Overview of methionine synthesis. (A) The standard pathway with 5-methyl-THF. (B) The reaction catalyzed by the core methionine synthases MesA and MesB. (C) The structure of methylcobalamin. Cobalamin has 5,6-dimethylbenzimidazole as the lower ligand, but many organisms use cobamides with other lower ligands. (D) The structure of 5-methyl-THF. Although THF is shown with a single glutamyl residue (at right), in the cell, THF is usually polyglutamylated.

Besides MetH and MetE, three other types of methionine synthases have been reported. These "core" methionine synthases (Deobald et al. 2020) are homologous to the C-terminal catalytic 
domain of MetE and do not contain any other domains. In particular, they lack the N-terminal domain of MetE that is involved in binding folate (Pejchal and Ludwig 2005). We describe each of these families below.

First, a core methionine synthase from the methanogen Methanobacterium thermoautotrophicum has been studied biochemically (Schröder and Thauer 1999). We will call this protein MesA (methionine synthase $\underline{A}$; UniProt:METE_METTM). In vitro, MesA transfers methyl groups from methylcobalamin to homocysteine to form methionine (Figure 1), but it has no activity with 5-methyl-THF or 5-methyltetrahydromethanopterin as substrates (Schröder and Thauer 1999). (Tetrahydromethanopterin is a cofactor for methanogenesis that is similar to THF.) Because MesA has a very weak affinity for methylcobalamin (the Michaelis-Menten constant is above $20 \mathrm{mM}$ ), and because most of the cobalamin in methanogens is bound to corrinoid proteins, the physiological substrates of MesA are probably methyl corrinoid proteins (Schröder and Thauer 1999). It might seem surprising that MesA accepts methyl groups from cobamides when it is homologous to the cobalamin-independent enzyme MetE, but the catalytic mechanisms of MetE and MetH are similar: both MetE and MetH rely on a zinc cofactor to deprotonate the sulfur atom of homocysteine and activate it as a nucleophile (Matthews et al. 2003).

Second, a core methionine synthase from the bacterium Dehalococcoides mccartyi was recently identified (Deobald et al. 2020). We will call this protein MesB (UniProt:A0A0V8M4G6). MesB uses methylcobalamin, but not 5-methyl-THF, as a substrate in vitro, and MesB cannot complement a metE- strain of E. coli (Deobald et al. 2020). MesB was proposed to obtain methyl groups from the iron-sulfur corrinoid protein (CoFeSP) of the Wood-Ljungdahl pathway (Deobald et al. 2020).

Third, a genetic study identified an unusual methionine synthase in Acinetobacter baylyi ADP1 (de Berardinis et al. 2008). ACIAD3523 (UniProt:Q6F6Z8) is required for methionine synthesis in the absence of cobalamin, and so is the adjacent gene ACIAD3524. Although ACIAD3523 was originally annotated as a MetE protein and is so described in the genetic study, ACIAD3523 lacks the N-terminal folate-binding domain, and it is distantly related to the C-terminal catalytic domain of MetE (under 30\% identity). The associated protein ACIAD3524 belongs to the uncharacterized family DUF1852 (Pfam PF08908, (Finn et al. 2014); DUF is short for domain of unknown function.) Homologs of ACIAD3523 are found adjacent to genes encoding DUF1852 in diverse bacteria; we will call these families MesD and MesX.

These three types of core methionine synthases are distantly related to each other (under $30 \%$ pairwise amino acid identity), so we used them to define families and analyzed their distribution across bacteria and archaea. We also noticed that several genera of anaerobic archaea lack all known forms of methionine synthase, but grow in minimal media. These archaea contain another family of putative core methionine synthases, with conserved functional residues, which we call MesC. 
We found that MesA is found solely in methanogens. MesB and MesC are found solely in organisms with the Wood-Ljungdahl pathway, so we propose that most members of both families obtain methyl groups from CoFeSP. MesD is found solely in aerobic bacteria. By using pooled mutant fitness assays and complementation assays, we will show that 5-methyl-THF is not the methyl donor for MesD, that MesD requires both MesX and oxygen for activity, and that MesD and MesX suffice to convert homocysteine to methionine in E. coli.

\section{Results and Discussion}

\section{Identification of split MetE proteins and the MesC family}

We previously ran the GapMind tool for reconstructing amino acid biosynthesis against 150 genomes of bacteria and archaea that grow in defined minimal media without any amino acids present (Price et al. 2020). After updating our analysis to account for MesB and MesD, six archaeal genomes lacked candidates for any of the known types of methionine synthase (MetH, MetH split into two or three parts (Price et al. 2018a; Price et al. 2020), MetE, MesA, MesB, or MesD).

We searched for additional candidates for methionine synthase by using the profile of protein family COG0620 (Tatusov et al. 2003) and PSI-BLAST (Altschul et al. 1997). COG0620 matches both the $\mathrm{N}$-terminal (folate-binding) and C-terminal (homocysteine-activating) domains of MetE, as well as MesA, MesB and MesD.

In the hyperthermophilic archaea Pyrolobus fumarii 1A, we identified two hits to COG0620, which correspond to the two domains of MetE. PYRFU_RS09465 contains the N-terminal domain (Meth_synt_1 in Pfam) and PYRFU_RS01495 contains the C-terminal domain (Meth_synt_2 in Pfam). The homologs of these proteins from Pyrococcus furiosus (PF1268 and PF1269, respectively) are encoded adjacent to each other and form a complex (Menon et al. 2009), which suggests that they comprise a split MetE. Split MetE proteins are found primarily in archaea, and they appear to be the sole form of methionine synthase in most of the thermophilic or halophilic archaea (see Methods). The two parts of split MetE are encoded adjacent to each other in diverse archaea and in the bacterium Sulfobacillus sp. hq2. This confirms that these proteins form a conserved system. Many of the previously-proposed "core" methionine synthases from archaea (see the first figure of (Deobald et al. 2020)) are probably catalytic subunits of split MetE proteins; this includes representatives from the genera Acidianus, Aeropyrum, Haloferax, Natronomonas, Pyrobaculum, Pyrococcus, and Sulfolobus. Given the domain content of split MetE proteins, we predict that they use methyl-THF or other methyl pterins as their methyl donors. (Thermophilic archaea are thought to use alternate pterins, instead of tetrahydrofolates, as their one-carbon carriers (de Crécy-Lagard et al. 2012).)

The other five archaea with missing methionine synthases were strict anaerobes: three strains of methanogenic Methanosarcina, an iron-reducing Ferroglobus placidus, and a sulfite-reducing 
Archaeoglobus veneficus. All genomes contained one or more putative core methionine synthases, such as MA_0053 (UniProt:Q8TUL3). These proteins were similar to each other (33\% identity or above) and were more distantly related to MesA, MesB, or MesD (pairwise identities under $30 \%$ ). We will call them MesC.

\section{The MesA family is found only in methanogens}

Given the four types of core methionine synthases that we are aware of, we searched for likely functional orthologs of each, and we examined their distribution across 7,694 bacteria and 321 archaea from UniProt's reference proteomes (UniProt Consortium 2019). We will discuss MesA first.

Although MesA is distantly related to the other types of core methionine synthases, MesA is similar to split MetE proteins. For instance, the characterized MesA is $38 \%$ identical to the putative catalytic component of the split MetE from Pyrolobus fumarii. We considered closer homologs to form the MesA family. Using phmmer from the HMMer package (http://hmmer.org/), we found 40 such hits ( $40 \%$ identity or above). All of these putative MesA proteins were from methanogens: this included representatives of 18 genera from the orders Methanobacteriales, Methanocellales, Methanococcales, Methanomicrobiales, and Methanopyrales.

Although most of the methanogens that have MesA encode the Wood-Ljungdahl pathway, some do not, including several species of Methanobrevibacter (such as M. curvatus). This suggests that the CoFeSP is not the physiological methyl donor for MesA. Also, some methanogens contain both MesA and MesB, which suggests that the two methionine synthases might use different methyl donors. To identify potential methyl donors for MesA, we examined the protein families that were reported to be universally conserved in methanogens (Basu et al. 2011). As far as we know, only two of these families are thought to bind cobamides: Mmp10 (methanogenesis marker protein 10) and MtrA. Mmp10 is a S-adenosylmethionine-dependent methyltransferase (Radle et al. 2019); since methionine is the precursor to S-adenosylmethionine, Mmp10 cannot be the source of methyl groups for methionine synthesis. MtrA is the corrinoid subunit of methyltetrahydromethanopterin:coenzyme M methyltransferase (Harms and Thauer 1996), which catalyzes the last methyl transfer reaction before reduction to methane. We predict that MesA obtains methyl groups from MtrA.

\section{The MesB family is linked to the iron-sulfur corrinoid protein of the Wood-Ljungdahl pathway}

To identify likely functional orthologs of MesB, we began with the hypothesis (Deobald et al. 2020) that MesB obtains methyl groups from the CoFeSP protein of the Wood-Ljungdahl pathway (Figure 2). This hypothesis is consistent with labeling experiments with Dehalococcoides mccartyi, which show that the methyl group of methionine is derived from the methyl group of acetate (Zhuang et al. 2014). It also explains how D. mccartyi can biosynthesize methionine despite encoding neither methylene-THF reductase (MetF), which is how most 
bacteria form methyl-THF, nor AcsE, which transfers methyl groups between CoFeSP and methyl-THF.

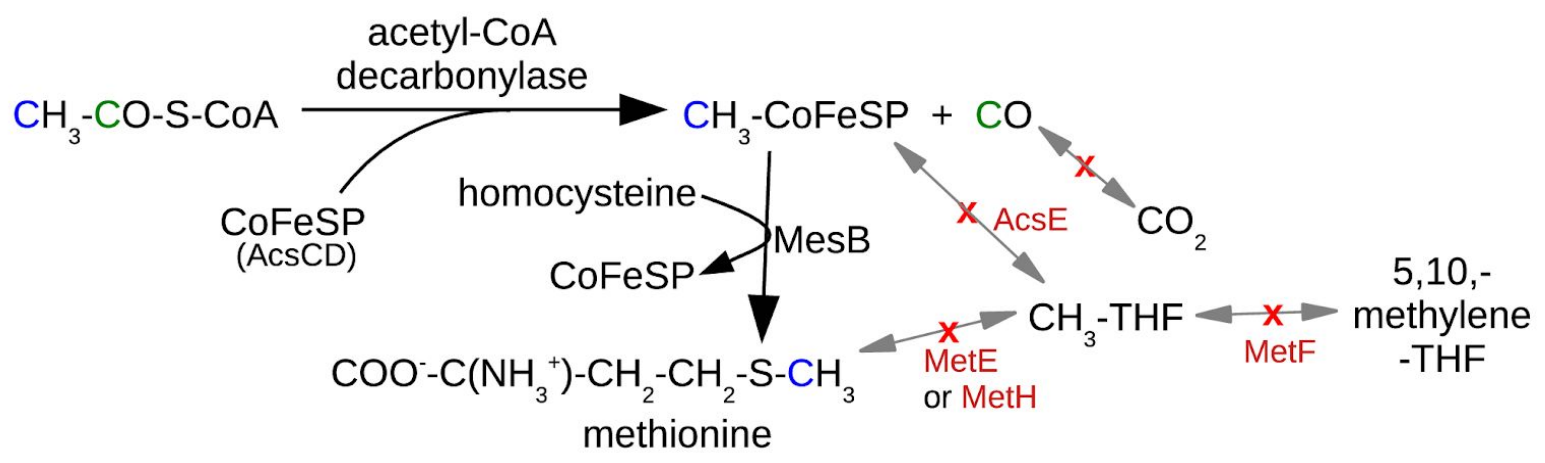

Figure 2: The proposed pathway for methionine synthesis in Dehalococcoides mccartyi. Steps that are absent from $D$. mccartyi are shown with a red $\mathrm{x}$.

Given this hypothesis, we selected homologs of MesB from MicrobesOnline (Dehal et al. 2010), we built a phylogenetic tree with MUSCLE (Edgar 2004) and FastTree 2 (Price et al. 2010), and we checked for the presence or absence of the Wood-Ljungdahl pathway in the organisms that contain close homologs. As shown in Figure 3A, most of the close homologs of MesB are found in organisms with the Wood-Ljungdahl pathway. But there are also close homologs of MesB in some anoxygenic phototrophic Chloroflexales (Chloroflexus or Roseiflexus) that lack the Wood-Ljungdahl pathway. As discussed below, the homologs from Chloroflexales lack the residues required for catalysis. We propose that the homologs of MesB in Chloroflexales have another function, while the homologs in Wood-Ljungdahl organisms are MesB-type core methionine synthases. 
A

Tree of MesB and related proteins

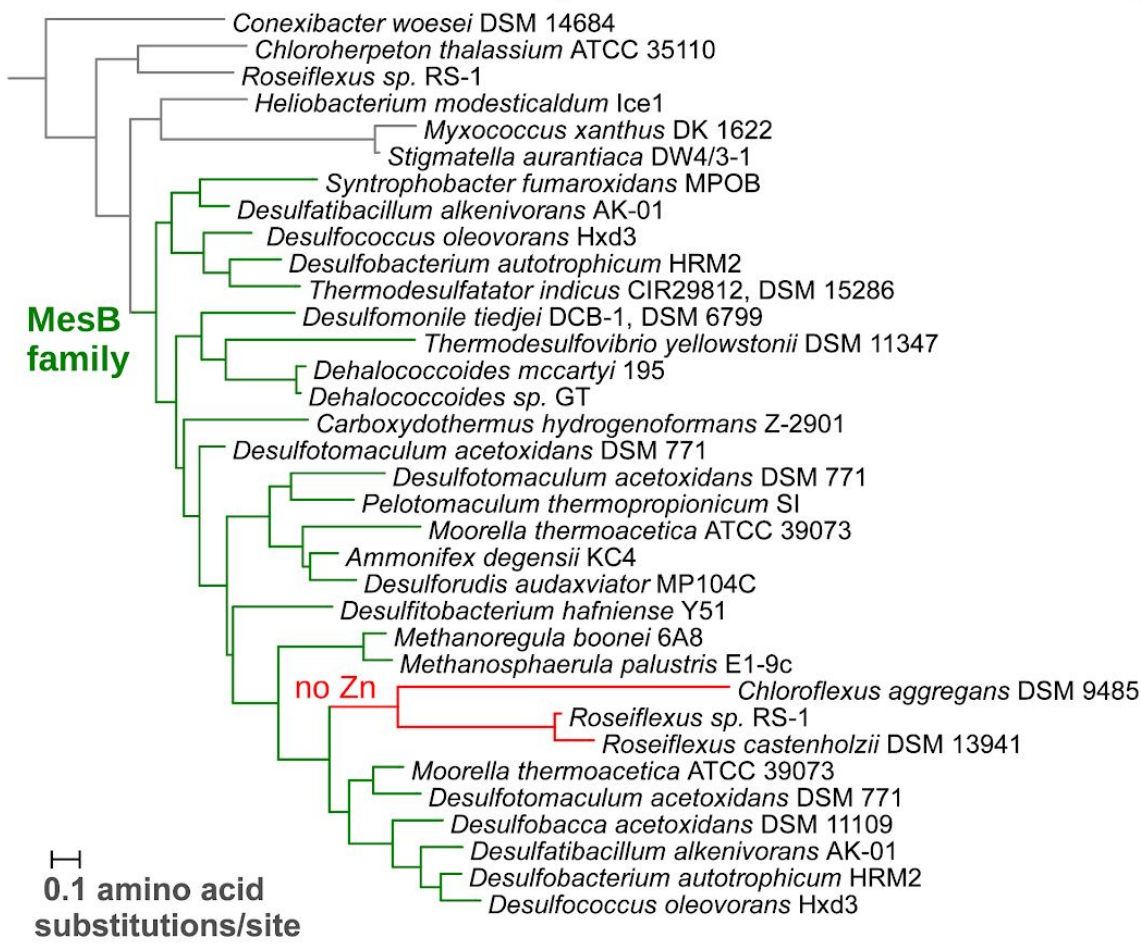

\section{Presencelabsence of other genes}
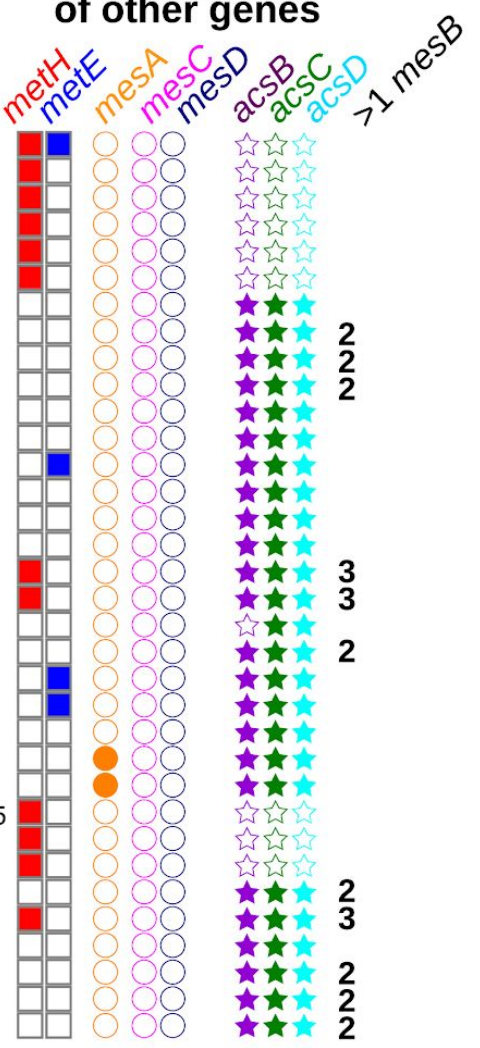

B

\begin{tabular}{|c|c|c|c|c|c|}
\hline $\begin{array}{c}\text { acsB } \\
\text { (acetyl-CoA } \\
\text { decarbonylase) }\end{array}$ & $\begin{array}{c}a c s D \\
\text { (CoFeSP } \\
\text { subunit) }\end{array}$ & $\begin{array}{c}\text { acsC } \\
\text { (CoFeSP } \\
\text { subunit) }\end{array}$ & $\begin{array}{c}\text { RACo } \\
\text { (activation of } \\
\text { CoFeSP) }\end{array}$ & mes $B$ & $\begin{array}{l}\text { acsF } \\
\text { (nickel insertion } \\
\text { into AcsB?) }\end{array}$ \\
\hline 1196 & 1195 & 1194 & Mboo_1193 & 1192 & 1191. \\
\hline
\end{tabular}

Methanosphaerula palustris E1-9c

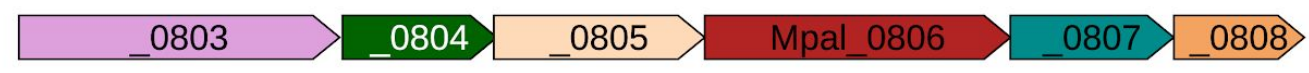

\section{Figure 3: Comparative genomics links MesB to the Wood-Ljungdahl pathway. (A) A}

phylogenetic tree of MesB and related proteins. The MesB family is highlighted in green and a subfamily that lacks $\mathrm{Zn}$-coordinating residues is highlighted in red. On the right, filled symbols indicate the presence in that genome of other methionine synthases or of the Wood-Ljungdahl pathway (acsBCD, also known as cdhCED). If the genome contains more than one mes $B$ gene, we show the number. The tree and the genome properties were rendered with iTOL v5 (https://itol.embl.de/). (B) Conserved clustering of mesB with genes from the Wood-Ljungdahl pathway. Gene drawings were modified from MicrobesOnline (Dehal et al. 2010). 
To test more broadly if MesB is present in any organisms that lack the Wood-Ljungdahl pathway, we used phmmer to examine the UniProt reference proteomes. We found 173 hits for MesB with at least 130 bits ( $29 \%$ identity or above). After excluding the non-enzymatic homolog from Roseiflexus sp. RS-1 and related proteins from two other Chloroflexi, we had 170 putative MesB proteins from 123 proteomes. All of these proteomes contained AcsC or AcsD, which are the two subunits of CoFeSP, and almost all (120/123) contained both AcsC and AcsD.

Many of the bacteria with putative MesB proteins lack the other types of methionine synthase (Figure 3A). Also, many bacteria that contain MesB as the sole putative methionine synthase are known to grow in minimal media. For instance, among the prototrophic bacteria that we previously analyzed with GapMind (Price et al. 2020), MesB appears to be the sole methionine synthase in representatives of the genera Desulfacinum, Desulfallas, Desulfarculus, Desulfatibacillum, Desulfatiglans, Desulfitobacterium, Desulfobacca, and Thermodesulforhabdus. These observations strongly suggest that these homologs of MesB are methionine synthases. In contrast, more distant homologs of MesB are found in bacteria that encode other methionine synthases, which suggests that these MesB-like proteins might have another function.

Although MesB is primarily found in bacteria, it is also found in some methanogens, many of which contain MesA as well. In some methanogens, the mesB genes are in an apparent operon with acs $C$ and $a c s D$, which encode the two subunits of CoFeSP protein (Figure 3B). This conserved operon suggests a direct functional relationship.

The distribution of mes $B$, as well as the gene neighborhood of mes $B$ in methanogens, suggest that most of the MesB proteins use the CoFeSP protein from the Wood-Ljungdahl pathway as the methyl donor. Some of the bacterial genomes with mes $B$ contain 2 or 3 members of the family (Figure $3 \mathrm{~A}$ ), and some of these paralogs might bind another corrinoid protein.

\section{The MesC family is found in archaea with the Wood-Ljungdahl pathway}

All of the organisms that we initially discovered MesC in are anaerobic archaea that encode the Wood-Ljungdahl pathway. To examine the distribution of MesC in the reference proteomes, we used phmmer with Q8TUL3 from Methanosarcina acetivorans as the query. We found a weak hit of MesC to protein A0A166AZ17 from Methanobrevibacter curvatus, which does not contain AcsC or AcsD. A0A166AZ17 is more similar to MesA (METE_METTM; 47\% identity instead of $22 \%$ identity), so we disregarded this hit. The other hits (38 bits or higher) were all to anaerobic archaea that contain AcsC and AcsD, except for the uncultured archaeal lineage MSBL-1. MesC was found in nine draft genomes of MSBL-1, and AcsC and/or AcsD were found in five of these nine. The Wood-Ljungdahl pathway is reported to be present in many MSBL-1 genomes (Mwirichia et al. 2016). Because these are incomplete single-cell genomes, we are not sure if the Wood-Ljungdahl pathway is truly absent from some of the MSBL-1 genomes that encode MesC-like proteins. 
Overall, MesC is found in archaea with the Wood-Ljungdahl pathway. This includes methanogens from the orders Methanosarcinales and Methanotrichales, iron-reducing Ferroglobus and Geoglobus, and sulfate-reducing or sulfite-reducing Archaeoglobus. We predict that most MesC proteins accept methyl groups from CoFeSP.

Although most of these organisms have just one methionine synthase, representatives of the genus Methanosarcina have 2-3 copies of MesC (and no other methionine synthases). The multiple MesC proteins within Methanosarcina seem to have arisen by lineage-specific duplications: they cluster together in a phylogenetic tree, and two of the three paralogs are near each other in the genome. Methanosarcina can grow on many different methylated compounds via an array of specialized corrinoid proteins (Fu and Metcalf 2015), so we speculate that some paralogs of MesC accept methyl groups from other corrinoid proteins besides CoFeSP.

It's interesting to consider the prevalence of the different types of methionine synthase across the methanogens. The Methanosarcinales have MesC only, while most other orders of methanogens have MesA only (this includes Methanobacteriales, Methanocellales, Methanococcales, most Methanomicrobiales, and Methanopyrales). Some Methanomicrobiales have both MesA and MesB. The Methanomassiliicoccales have MesB only; because the Methanomassiliicoccales have the Wood-Ljungdahl pathway and lack methyltetrahydromethanopterin:coenzyme M methyltransferase (Borrel et al. 2016), this is consistent with our predictions. The extremely halophilic methyl-reducing methanogens Methanonatronarchaeum thermophilum and Methanohalarchaeum thermophilum (Sorokin et al. 2018) contain split MetE proteins but not other types of methionine synthases. We did not identify MetH or MetE in any methanogens, possibly because most methanogens lack 5-methyl-THF (although 5-methyl-THF might be present in Methanosarcina (Buchenau and Thauer 2004)).

\section{The MesD family is found in diverse aerobic bacteria}

To examine the distribution of the core methionine synthase MesD and the associated protein MetX (DUF1852), we first used MicrobesOnline to identify homologs of ACIAD3523 (MesD) that are likely to have the same function in methionine synthesis. Specifically, we used the MicrobesOnline tree-browser to check if they were adjacent to DUF1852. We chose a BLASTp bit score threshold of 390 bits ( $55 \%$ identity), as homologs above this threshold were almost always adjacent to DUF1852. We also excluded a homolog from the oomycete Phytophthora capsici, which could be contamination or might indicate the acquisition of DNA from a Stenotrophomonas bacterium. This left 106 genomes from 44 genera that contain MesD. We used FAPROTAX (Louca et al. 2016) and web searches to check the lifestyles of these genera and found that all 44 of them were aerobic. These include both strict aerobes and facultative anaerobes. 
To check more broadly that MesX is found only in aerobic bacteria, we used AnnoTree (Mendler et al. 2019) to obtain a list of 235 genera that contain the corresponding Pfam (PF08908). After removing suffixes (i.e., converting "Erythrobacter_B" to "Erythrobacter"), we found MesX in 206 named genera, of which 170 were distinct from the genus names in MicrobesOnline. We examined a random sample of 50 of these 170 genera and all were aerobic.

Finally, we ran phmmer against UniProt reference proteomes with ACIAD3523 as the query. We considered homologs with a score of at least 560 bits ( $72 \%$ identity) to be MesD proteins. Almost all of the proteomes with putative MesD proteins (395/399) contained MesX (DUF1852) as well. Organisms with both MesD and MesX included representatives of the $\alpha$-Proteobacteria, $\beta$-Proteobacteria, $Y$-Proteobacteria, Actinobacteria, Bacteroidetes, and Verrucomicrobia. Genomes with MesD/MesX often contain MetH ( $75 \%$ of cases) or MetE ( $32 \%$ of cases) or both ( $29 \%$ of cases), and MesD/MesX appears to be the sole methionine synthase in just $22 \%$ of the organisms that have it. We speculate that MesD/MesX co-occurs with the other methionine synthases because MesD/MesX is only active under aerobic conditions.

\section{Conserved functional residues of core methionine synthases}

To check if the MesA, MesB, MesC, and MesD families have conserved functions, we examined functional residues involved in catalysis and in binding homocysteine. First, MetE activates homocysteine via a zinc thiolate intermediate, and the residues that coordinate the zinc atom in E. coli's MetE are His641, Cys643, Glu665, and Cys726 (Taurog and Matthews 2006). The characterized MesB protein (Deobald et al. 2020) has an aspartate instead of Glu665; this substitution appears to be compatible with zinc binding and catalytic activity.

The zinc-binding residues are highly conserved in the MesA, MesB, and MesD families (Figure 4). The only exceptions are two MesB proteins that have an asparagine instead of Cys726; this might still be compatible with zinc binding. In the MesB-like proteins from Chloroflexales (shown in red in Figure 3A, and not included in Figure 4), the zinc-binding residues are not conserved: instead of HCEC or HCDC, they have FSHG, YCDQ, or YREQ.

Most MesC proteins have a histidine instead of Cys643 (Figure 4), which is likely to be compatible with zinc binding. Two MesC proteins from Methanosarcina have a tyrosine aligning to Cys643, and we are not sure if this is compatible with zinc binding and methyltransferase activity. However, these genomes include other representatives of $\mathrm{MesC}$, and those other proteins do have histidines aligning to Cys643. All eight MesC proteins from the uncultured lineage MSBL-1 had a glycine aligning to Glu665, which we would not expect to be compatible with zinc binding. The MSBL-1 genomes contain likely split MetE proteins (i.e., AKJ63_00345:AKJ63_00350), so the MesC proteins from this lineage might have a different function. 


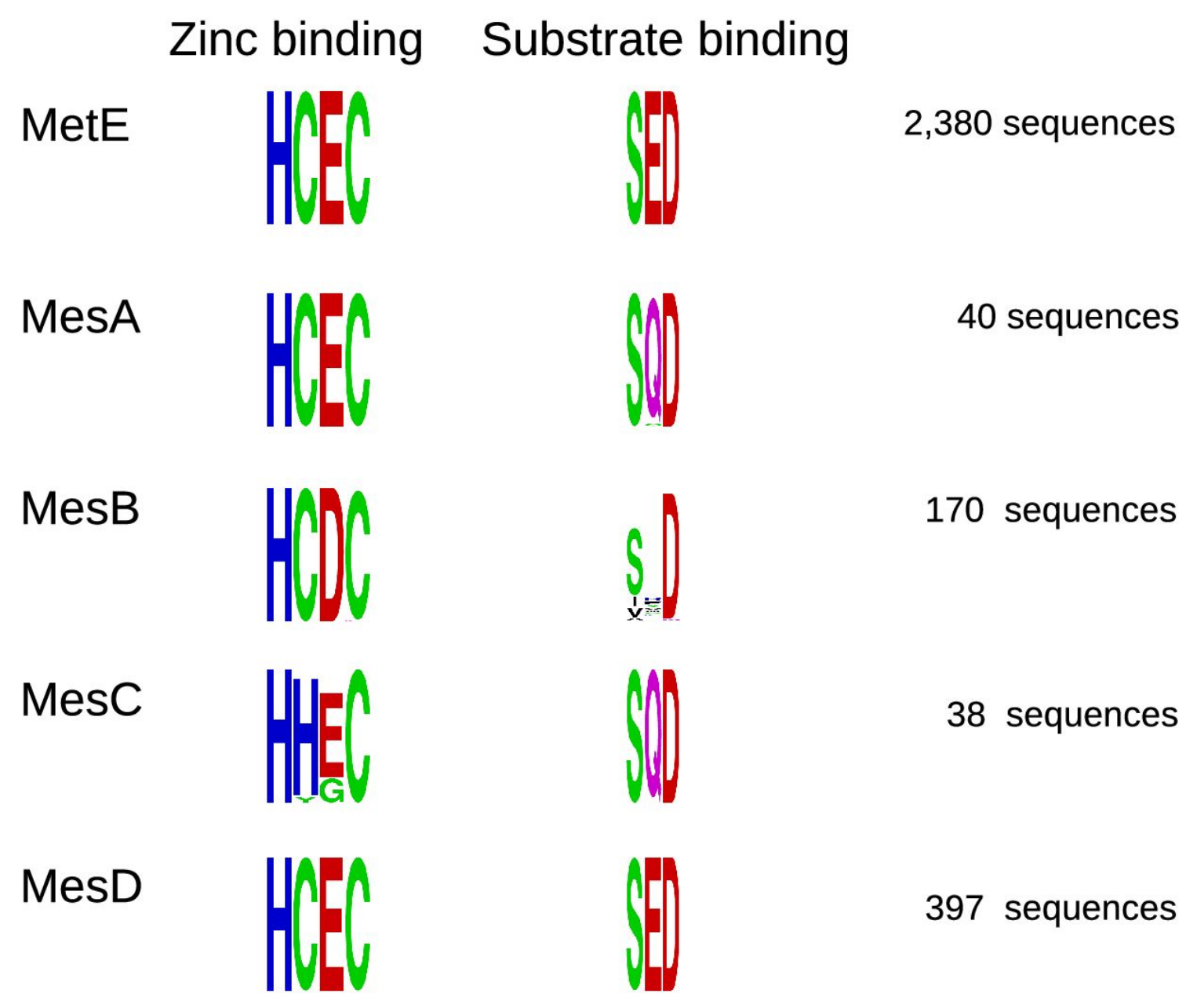

Figure 4: Functional residues of MetE and of core methionine synthases. We show sequence logos (Crooks et al. 2004) for the zinc-coordinating and substrate-binding residues of each family of methionine synthases. The height of each position shows its conservation within the family, as measured by information content or bits. In MetE from E. coli, the zinc-coordinating residues are $\mathrm{H} 641, \mathrm{C} 643, \mathrm{E} 665$, and $\mathrm{C} 726$, and the substrate-binding residues are S433, E484, and D599.

We then examined the substrate-binding residues. Structural data suggests that several side chains in MetE form hydrogen bonds with the amino or carboxyl groups of homocysteine or methionine (PDB:1U1J; (Ferrer et al. 2004)). (In the E. coli residue numbering, Ser433 binds the carboxyl group and Glu484 and Asp599 bind the amino group.) Similarly, in a crystal structure for a MesD protein bound to the methionine analog selenomethionine (PDB:3RPD), the corresponding side chains are in proximity to the amino and carboxyl groups of selenomethionine (Ser22, Glu73, and Asp188 in 3RPD). As shown in Figure 4, MesA and MesC have similar residues, but with a glutamine instead of Glu484. Glutamine could also form a 
hydrogen bond with the amino group of homocysteine, so we predict that MesA and MesC bind homocysteine (or methionine) in the same manner that MetE and MesD do. The identity of these residues between MesA and MesC supports our prediction that MesC is also a methionine synthase. In MesB, Ser433 and Asp599 are mostly conserved, but Glu484 is not. The region corresponding to Glu484 (around Trp69 in the characterized MesB) is quite variable among MesB proteins and is difficult to align to MetE. Overall, we found that the functional residues for binding zinc and homocysteine were conserved in all four families of core methionine synthases.

\section{MesD requires MesX and oxygen, but not 5-methyl-THF}

We then investigated the function of MesD and MesX in more detail. In particular, we wondered what the source of methyl groups is for MesD. If MesD accepts methyl groups from 5-methyl-THF, then the methylene-THF reductase (MetF) would be required for its activity. But we have several pieces of evidence that MetF is not required for MesD's activity.

First, in Acinetobacter baylyi, mes $D$ and mes $X$ are required for growth if neither methionine nor vitamin B12 are available (de Berardinis et al. 2008). ( $A$. baylyi also has a cobalamin-dependent methionine synthase (MetH), but MetH is probably not active under these conditions because $A$. baylyi cannot synthesize cobalamin.) In a constraint-based metabolic model of $A$. baylyi in which 5-methyl-THF is a precursor to methionine, metF is predicted to be essential for growth (Durot et al. 2008), which illustrates that MetF is the only known path to 5-methyl-THF. Nevertheless, metF from $A$. baylyi is not essential for growth in a defined minimal medium with no vitamins (de Berardinis et al. 2008). This suggests that MesD/MesX do not require methyl-THF. A caveat is that another protein from $A$. baylyi, ACIAD1783, is distantly related to MetF, but ACIAD1783 lacks the N-terminal part of MetF and probably has another function (see Methods).

Second, although most of the organisms with MesD also contain MetH or MetE, we did find 85 proteomes in which MesD seems to be the sole methionine synthase. Many of these proteomes $(69 \%)$ seem to lack MetF. One of them is Paenarthrobacter aurescens TC1, which can grow in a defined minimal medium with the herbicide atrazine as the sole source of carbon (Strong et al. 2002). (This species was formerly known as Arthrobacter aurescens.) The degradation pathway for atrazine does not involve 5-methyl-THF or other folate derivatives (Sajjaphan et al. 2004). Again, it appears that 5-methyl-THF is not required for MesD's activity.

Third, we used high-throughput genetics to investigate methionine biosynthesis in Sphingomonas koreensis DSMZ 15582. The genome of $S$. koreensis encodes mes D, mes $X$, and $m e t H$ (split into two parts), but not metE or genes for the de novo biosynthesis of cobamides (Shelton et al. 2019). If cobalamin is not provided in the media, mes $D$ should be required for methionine synthesis. We grew a pool of over 250,000 barcoded transposon mutants of S. koreensis (Price et al. 2016) in defined media with no cobalamin and with cellobiose, glucose, or glutamate as the sole source of carbon. As shown in Figure 5, both mes $D$ and mes $X$ were important for fitness unless methionine was added. MetF was less 
important for fitness, especially when glutamate was the carbon source. We observed mild phenotypes for disrupting either part of the split $m e t H$, which might indicate some carry-over of vitamin B12 from the media used to recover the mutant pool from the freezer. (The recovery media contains tryptone, which is often made by hydrolyzing animal protein.) Besides mes $D$ and mes $X$, most of the genes whose mutants were rescued by added methionine were involved in homocysteine biosynthesis or other metabolic processes that are not necessary if methionine is available (Figure S1). The few other genes whose mutants were rescued are not expected to be involved in methionine biosynthesis (Figure S1). Overall, in S. koreensis, mes $D$ and mesX were required for methionine biosynthesis in the absence of cobalamin, but metF was not.

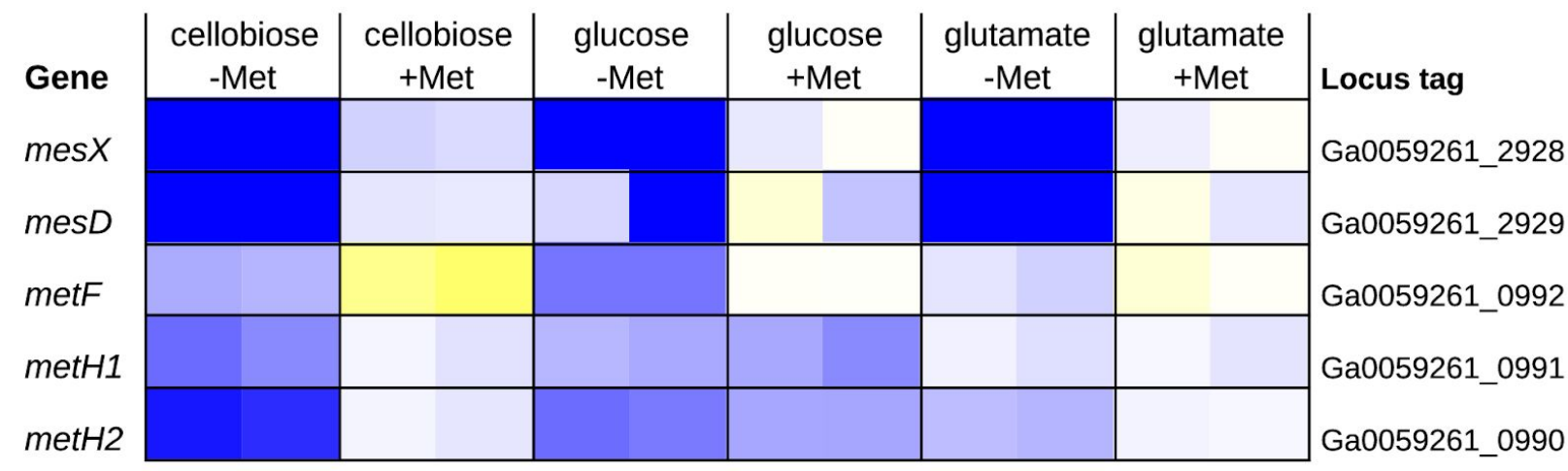

\section{Gene fitness \\ $=\log _{2}\left(\begin{array}{c}\text { change in relative } \\ \text { abundance }\end{array}\right)$}

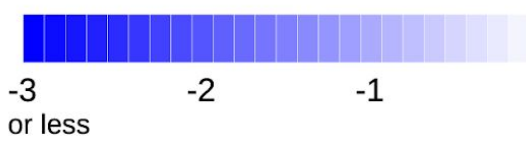

important for fitness
0

no effect
$+1$

$+2$

or more

detrimental

Figure 5: Sphingomonas koreensis can grow in minimal media by using MesD and not MetF. A pool of transposon mutants was grown in a defined minimal media with a single carbon source and without added vitamins. Some cultures were supplemented with $250 \mu \mathrm{M}$ L-methionine. Each cell in the heatmap shows a gene fitness value from a different experiment; each condition has two replicates. A gene fitness value is the $\log _{2}$ change in the relative abundance of mutants in that gene during that experiment (from inoculation at $\mathrm{OD}_{600}=0.02$ until saturation).

Finally, we cloned mes $D$ and mes $X$ from $S$. koreensis into various strains of $E$. coli. $E$. coli encodes both metE and metH, but in the absence of cobalamin, metE is required for methionine biosynthesis. We obtained strains of $E$. coli with metE or metF deleted from the Keio collection (Baba et al. 2006). During aerobic growth in minimal glucose M9 medium, which lacks cobalamin, the wild-type (parent) strain grows, but neither $\Delta m e t E$ nor $\Delta m e t F$ strains grow (Figure 6). Growth of either $\Delta$ metE or $\Delta$ metF strains was rescued when both mes $D$ and mes $X$ were provided on a plasmid, but not when mes $D$ or mes $X$ were provided individually (Figure 6). This confirms that MesD is a methionine synthase that requires MesX, but not MetF, for activity. 


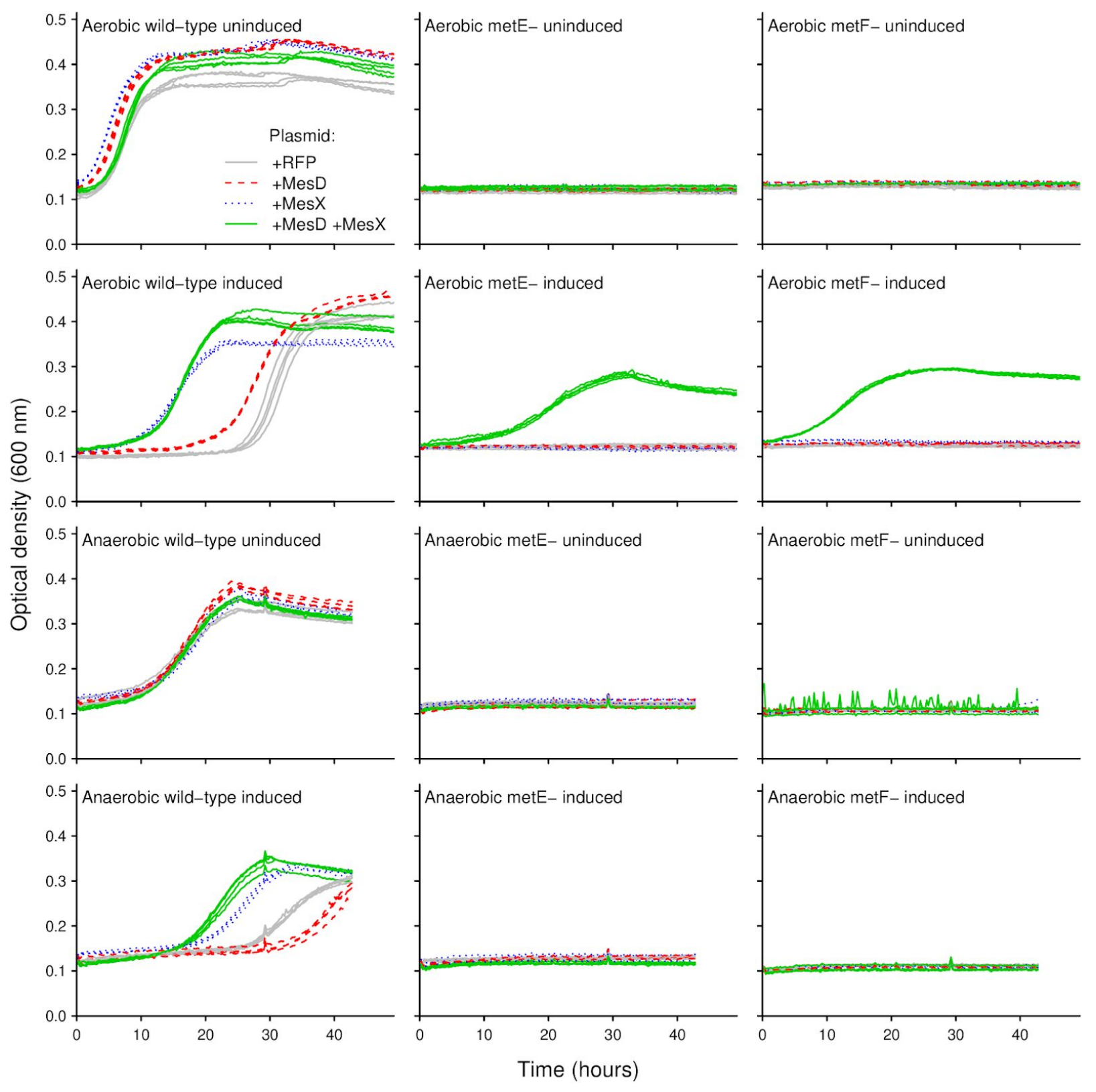

Figure 6: Complementation assays show that MesD requires MesX and oxygen for activity, but not MetF. We cloned MesD, MesX, or MesD and MesX together into strains of $E$. coli from the Keio collection (Baba et al. 2006) and measured growth in minimal glucose M9 medium. A plasmid bearing red fluorescent protein (RFP) was used as a control.

When we repeated these complementation assays under anaerobic conditions, we found that mes $D$ and mes $X$ could no longer rescue the growth of $\Delta$ metE or $\Delta m e t F$ strains. Given this data and the phylogenetic distribution of MesD/MesX, we conclude that MesD/MesX can only function under aerobic conditions. In principle, this could reflect interactions with the electron transport chain, such as a requirement for ubiquinone. But since neither MesD nor MesX are expected to be membrane proteins (Sonnhammer et al. 1998), we predict that MesD/MesX 
require molecular oxygen (or perhaps hydrogen peroxide, which is produced by respiring cells) for activity.

\section{What is the molecular function of MesX?}

MesD does not obtain methyl groups from 5-methyl-THF: it lacks the N-terminal folate-binding domain of MetE, and metF is not required for MesD's activity. Instead, we predict that MesX provides methyl groups to MesD, either by covalently binding the methyl group, or by forming a methylated substrate that MesD can act on. This would also explain why mes $X$ is required for methionine synthesis in Acinetobacter baylyi and Sphingomonas koreensis and why mesD alone is not sufficient for methionine synthesis in E. coli.

The requirement for oxygen implies that MesX oxidizes its substrate. Furthermore, MesD and MesX are often encoded near a putative flavin reductase (i.e., ACIAD3522 or Ga0059261_2931); this also suggests that MesX obtains methyl groups by a redox reaction. If MesX is an oxidase, then it cannot obtain methyl groups from other folate compounds such as 5,10-methylene-THF, which would need to be reduced. Because MesD/MesX are found in diverse bacteria and can function in E. coli, we predict that MesX obtains methyl groups from central metabolism. As a purely illustrative example, an oxygenase reaction with pyruvate and a reduced flavin could form hydrogen peroxide, glyoxylate, a methyl group attached to a nitrogen or sulfur atom in a side chain of MesX, and oxidized flavin.

\section{Conclusions}

We analyzed four families of core methionine synthases. Based on their distributions, we predicted that MesA obtains methyl groups from the MtrA protein of methanogenesis, while MesB and MesC obtain methyl groups from the CoFeSP protein of the Wood-Ljungdahl pathway. In contrast to MesA, MesB, and MesC, which are found solely in strictly anaerobic organisms and probably accept methyl groups from corrinoid (cobamide-binding) proteins, MesD is found solely in aerobic organisms, and MesD does not require vitamin B12 or other cobamides as cofactors. We showed that MesD requires MesX (DUF1852) for activity, and that this system functions in $E$. coli, but only under aerobic conditions. We predict that MesD/MesX obtains methyl groups from central metabolism in an oxygen-dependent process. It appears that diverse bacteria and archaea can synthesize methionine without 5-methyltetrahydrofolates or other methyl pterins as intermediates.

\section{Materials and Methods}

\section{Literature searches}

Literature on MetE and related proteins was retrieved using PaperBLAST (Price and Arkin 2017) and by using PaperBLAST's family search for PF01717 (the catalytic domain). 


\section{Phylogenetic profiling}

We downloaded the predicted protein sequences for 321 archaea and 7,694 bacteria from UniProt reference proteomes on October 13, 2020. We searched for MesA, MesB, MesC, MesD, MesX, AcsD/CdhD, AcsC/CdhE, MetE, MetH, MetF, and split MetE proteins. To find homologs of the protein sequences or models (listed below), we used phmmer or hmmsearch from HMMer 3.3.1. For searches with curated models, we used the trusted cutoff (--cut_tc). Otherwise, we used the bit score threshold given below, or else we used $E<0.001$. For MesA, we used hits of 174 bits or higher to the characterized protein (METE_METTM). For MesB, we used hits of 173 bits or higher to DET0516 from Dehalococcoides mccartyi, and we excluded three non-enzymatic homologs from Chloroflexi. (DET0516 is the MesB protein in D. mccartyi 195 , and is $99 \%$ identical to the characterized MesB protein from D. mccartyi CBDB1.) For MesC, we used hits of 38 bits or higher to Q8TUL3. For MesD, we used hits of 560 bits or higher to ACIAD3523. For MesX, we used model PF08908.11 from PFam. For AcsD/CdhD and AcsC/CdhE, we used representatives from Methanosarcina acetivorans C2A as queries (ACDD1_METAC and ACDG_METAC, respectively). To identify MetE, we used TIGR01371 from TIGRFam (Haft et al. 2013). To identify MetH, we used TIGR02082, but we supplemented these results. During a preliminary analysis of the bacteria that do not contain known forms of methionine synthase, we found that many of them actually contained homologs of the MetH protein from Thermotoga maritima (UniProt:Q9WYA5). Biochemical assays have confirmed that the protein from T. maritima is a methionine synthase (Huang et al. 2007), but it scores below the trusted cutoff of TIGR02082. We used protein BLAST with Q9WYA5 as the query to identify additional MetH proteins, with $\mathrm{E}<0.001$ and at least $80 \%$ coverage of the query. The identification of MetF and split MetE proteins are more complex and are described below.

\section{Searching for methylene-tetrahydrofolate reductase proteins}

To identify putative methylene-tetrahydrofolate reductases (MTHFR or MetF), we used model PF02219.1 from PFam. PF02219 is the only domain in characterized MetF proteins, and no other functions for the family have been reported. We also searched for the flavin-independent methylene-tetrahydrofolate reductases (Sah et al. 2020). These proteins are distantly related to MetF and do not have statistically significant hits to model PF02219.17. We used the iterative search tool jackhmmer (https://www.ebi.ac.uk/Tools/hmmer/search/jackhmmer) to find homologs of MSMEG_6596 in UniProt reference proteomes. (These preliminary searches were run in May 2020.) The first iteration found 93 hits, all within Actinobacteria; the second iteration found 114 hits; and the third iteration found 541 hits, including 298 proteins with hits to PF02219. We used this third model (at $E<0.001$ ) to identify additional methylene-tetrahydrofolate reductases in all of the UniProt reference genomes (as of October 2020).

For a few genomes of interest, we also used Curated BLAST for Genomes (Price and Arkin 2019) with 1.5.1.20 (the Enzyme Classification number for MetF) to try to find additional candidates. In Acinetobacter baylyi and Sphingomonas koreensis, we identified the proteins that 
are annotated as MetF, and we did not identify any proteins in Dehalococcoides mccartyi or Paenarthrobacter aurescens. But in A. baylyi, we also identified ACIAD1783, which is distantly related to characterized MTHFR proteins (under $30 \%$ identity) and lacks the $\mathrm{N}$-terminal part of PF02219. (It aligns to position 70-281 out of 287 in the model.) We are not aware of data about its function, but ACIAD1783 is 39\% identical to PP_4638 from Pseudomonas putida KT2440 along its full length. 101 fitness experiments for $P$. putida are available in the Fitness Browser (as of October 2020) and PP_4638 has no significant phenotypes. The fitness pattern for metF from $P$. putida (PP_4977) is virtually identical to that of the methionine synthase metH (PP_2375; $r=0.96$ ), which suggests that PP_4638 did not provide MTHFR activity in these growth conditions. Overall, ACIAD1783 could be a diverged MTHFR protein, but it probably has another function.

\section{Searching for split MetE proteins}

To identify the catalytic component of split MetE, we used phmmer with PF1269 from Pyrococcus furiosus (UniProt: METE_PYRFU) as the query, and a threshold of at least 200 bits, which excludes all representatives of MesA, MesB, MesC, MesD, and MetE. We kept only the highest-scoring candidate in each genome. The putative folate-binding component is more divergent and hence more challenging to identify; we used the Pfam model for the $\mathrm{N}$-terminal domain of MetE (Met_synt_1 or PF08267.12) and its trusted cutoff to identify candidates, but if a protein matched the catalytic domain (Met_synt_2 or PF08267.12) as well, then we required that the alignment for Met_synt_1 have the higher bit score. We also required that candidates for either component be at most 400 amino acids long. For comparison, members of the MesA, MesB, MesC, or MesD families were at most 386 amino acids, while the MetE proteins (matching TIGR01371) were at least 676 amino acids. Of 147 genomes with candidates for the catalytic component of split MetE, 107 (73\%) contain candidates for the putative folate-binding component; we considered these 107 genomes to contain split MetE proteins.

Our list of split MetE proteins is incomplete because the putative folate-binding component is difficult to identify. For instance, one of the top-ranking candidates for the catalytic component that was not accompanied by a putative folate-binding component was AMET1_0514 (UniProt: A0A1Y3GEY8) from Methanonatronarchaeum thermophilum. However, AMET_0514 is encoded adjacent to a protein that matches both Met_synt_1 and Met_synt_2 with similar scores (AMET1_0515; UniProt:A0A1Y3GF94), and was therefore excluded from the list of candidates for the folate-binding component.

We did not require that the two components of split MetE be encoded near each other in the genome, and there are a few genomes where they are not nearby (i.e., Pyrolobus fumarii). However, across diverse organisms, the two components were almost always adjacent to each other.

Of the 107 genomes with split MetE, 106 do not encode any of the other known forms of methionine synthase. (The exception was Haloquadratum walsbyi DSM 16790, which also 
encodes MetE.) Of the 106 genomes with split MetE as the sole methionine synthase, 73 are from the order Halobacteria of halophilic archaea and 20 are from the order Thermoprotei of thermophilic archaea. Except for MetE in $\mathrm{H}$. walsbyi, we did not identify any other form of methionine synthase in either Halobacteria or Thermoprotei.

\section{Analysis of MesB and its relatives}

To infer a phylogenetic tree of MesB and related proteins, we selected the 88 closest homologs of DET0516 in MicrobesOnline. We removed two truncated homologs and a highly diverged homolog (VIMSS11031200 from Mahella australiensis DSM 15567). Using the MicrobesOnline web site, the remaining 85 proteins were aligned using MUSCLE (Edgar 2004), and the alignment was trimmed to relatively-confident columns with Gblocks (Castresana 2000). We used a minimum block length of 2 and allowed at most half gaps at any position. A phylogenetic tree was inferred from the trimmed alignment with FastTree 2 and the JTT+CAT model (Price et al. 2010). Figure $3 A$ shows the proteins that are expected to be MesB (given the presence of the Wood-Ljungdahl pathway and functional residues) and their closest neighbors in the tree.

To identify the presence and absence of MetE in these genomes, we used TIGR01371; for MetH, we used COG1410; for MesA, we used BLASTp hits of 180 bits or higher to MTH775 (VIMSS 20772), which is over $90 \%$ identical to the characterized protein (METE_METTM); for MesC, we used BLASTp hits of 190 bits or higher to MA0053 (VIMSS 233378) from Methanosarcina acetivorans C2A; for MesD, we used BLASTp hits of 390 hits or higher to ACIAD3523 (VIMSS 590795). For AcsB, we used COG1614 (also known as CdhC). For AcsC, we used COG1456 (also known as CdhE). For AcsD, we used COG2069 (also known as CdhD).

\section{Aligning functional residues}

Substrate-binding residues were determined from PDB:1U1J (MetE from Arabidopsis thaliana in complex with zinc and methionine) and PDB:3RPD (MesD from Shewanella sp. W3-18-1 in complex with zinc and selenomethionine) using the ligand interaction viewer at rcsb.org and ligplot at PDBsum (Laskowski and Swindells 2011). We also used the structure-guided aligner MAFFT-DASH (Rozewicki et al. 2019) to align the C-terminal (catalytic) part of MetE (from A. thaliana and from E. coli) with MesD from Shewanella. Compared to 1U1J, 3RPD has an additional hydrogen bond involving the carboxylate group of selenomethionine and the side chain of Tyr226. Tyr226 is in a MesD-specific insertion that does not align with MetE or with other core methionine synthases, but Tyr226 is conserved within the MesD family.

To identify the corresponding residues in MesA, MesB, and MesC, we used MUSCLE (version 3.7 ) to align diverse sequences of these families (from MicrobesOnline) to the N-terminal part of MetE proteins. The corresponding residues of MesA from Methanothermobacter thermoautotrophicus (UniProt:O26869) were 216,218,240,301 (zinc-binding) and 8,59,174 (homocysteine-binding). The corresponding residues of MesB from Dehalococcoides mccartyi 195 (UniProt:Q3Z939) were 214,216,235,311 (zinc-binding) and 15,69,177 
(homocysteine-binding). The corresponding residues of MesC from Methanosarcina acetivorans (UniProt:Q8TUL3) were 204,206,225,314 (zinc-binding) and 11,59,166 (homocysteine-binding).

To align the representatives of MesA, MesB, MesC, and MesD from UniProt's reference proteomes, we ran MUSCLE separately for each family. To align the members of MetE, we used the model from TIGRFam (TIGR01371) and hmmalign from the HMMer package.

\section{Mutant fitness assays}

Mutant fitness assays used a pool of 260,291 randomly-barcoded transposon mutants of Sphingomonas koreensis DSMZ 15582 and were performed and analyzed as described previously (Price et al. 2018b). Briefly, the pool was recovered from the freezer in LB media at $30 \mathrm{C}$ until it reached log phase. It was then inoculated at $O D_{600}=0.02$ into a defined inorganic medium with no amino acids or vitamins and with either $20 \mathrm{mM}$ cellobiose, $5 \mathrm{mM}$ glucose, or 10 $\mathrm{mM}$ glutamate as the carbon source. The inorganic base medium contained $0.25 \mathrm{~g} / \mathrm{L}$ ammonium chloride, $0.1 \mathrm{~g} / \mathrm{L}$ potassium chloride, $0.6 \mathrm{~g} / \mathrm{L}$ sodium phosphate monobasic monohydrate, $30 \mathrm{mM}$ PIPES sesquisodium salt, and Wolfe's mineral mix (final concentrations of $0.03 \mathrm{~g} / \mathrm{L}$ magnesium sulfate heptahydrate, $0.015 \mathrm{~g} / \mathrm{L}$ nitrilotriacetic acid, $0.01 \mathrm{~g} / \mathrm{L}$ sodium chloride, $0.005 \mathrm{~g} / \mathrm{L}$ manganese (II) sulfate monohydrate, $0.001 \mathrm{~g} / \mathrm{L}$ cobalt chloride hexahydrate, $0.001 \mathrm{~g} / \mathrm{L}$ zinc sulfate heptahydrate, $0.001 \mathrm{~g} / \mathrm{L}$ calcium chloride dihydrate, $0.001 \mathrm{~g} / \mathrm{L}$ iron (II) sulfate heptahydrate, $0.00025 \mathrm{~g} / \mathrm{L}$ nickel (II) chloride hexahydrate, $0.0002 \mathrm{~g} / \mathrm{L}$ aluminum potassium sulfate dodecahydrate, $0.0001 \mathrm{~g} / \mathrm{L}$ copper (II) sulfate pentahydrate, $0.0001 \mathrm{~g} / \mathrm{L}$ boric acid, $0.0001 \mathrm{~g} / \mathrm{L}$ sodium molybdate dihydrate, and $0.003 \mathrm{mg} / \mathrm{L}$ sodium selenite pentahydrate). Some cultures were supplemented with $0.25 \mathrm{mM}$ L-methionine. These cultures were grown in a 24-well microplate and allowed to reach saturation. The abundance of each strain in each sample was measured by genomic DNA extraction, PCR amplification of barcodes, and sequencing on Illumina HiSeq. Gene fitness values were computed as described previously (Wetmore et al. 2015). Briefly, the fitness of a strain is the normalized log2 ratio of its (relative) abundance in the sample after growth versus in the sample before growth (i.e., at the time of transfer), and the fitness of a gene is the weighted average of the strain fitness values for insertions in that gene. Gene fitness values are normalized so that most values are near zero. All experiments met the previously-published metrics for biological consistency (Wetmore et al. 2015). The fitness data can be viewed in the Fitness Browser (http://fit.genomics.lbl.gov/, archived at https://doi.org/10.6084/m9.figshare.13172087.v1) and is archived in FigShare (https://doi.org/10.6084/m9.figshare.13146419.v1).

\section{Genetic complementation assays}

We cloned Ga0059261_2928 (mesX), Ga0059261_2929 (mesD), or the two genes together into $\mathrm{pBbA2c}$. This vector includes the origin of replication from plasmid $\mathrm{p} 15 \mathrm{a}$, a chloramphenicol resistance gene, the tet $R$ regulator, and the inducible $P_{\text {tet }}$ promoter (Lee et al. 2011). In pBbA2c-RFP (provided by Jay Keasling from the University of California, Berkeley), RFP is downstream of the inducible promoter, and cloning of target genes for overexpression replaces RFP. We used Phusion DNA polymerase and standard cycling conditions for all PCR reactions. 
Briefly, we linearized pBbA2c-RFP by PCR with oligonucleotides oAD232 and oAD233 and gel-purified this PCR product. (Oligonucleotide sequences are in Table 1.) Ga0059261_2928 (mesX) was PCR amplified from total S. koreensis genomic DNA with oAD793 and oAD794, Ga0059261_2929 (mesD) was amplified with oAD795 and oAD796, and both genes were amplified with oAD793 and oAD796. These inserts were cloned into the linearized and gel-purified pBbA2c using the Gibson assembly mastermix (New England Biolabs) following the manufacturer's instructions. Plasmids with the correct sequence were identified by Sanger sequencing. We then introduced these plasmids (and the pBbA2c-RFP control vector) by electroporation into three strains from the Keio gene deletion collection: the parental or wild-type strain (BW25113), the metE gene deletion strain, and the metF gene deletion strain (Baba et al. 2006). Transformants were selected on LB supplemented with $20 \mu \mathrm{g} / \mathrm{mL}$ chloramphenicol. We performed the complementation growth assays in 96-well microplates using M9 minimal media with $20 \mathrm{mM}$ D-glucose as the carbon source, $10 \mu \mathrm{g} / \mathrm{mL}$ chloramphenicol, and either with inducer (4 $\mathrm{nM}$ anhydrotetracycline) or without. (Besides glucose, the M9 medium contained $2 \mathrm{mM}$ magnesium sulfate, $0.1 \mathrm{mM}$ calcium chloride, $12.8 \mathrm{~g} / \mathrm{L}$ sodium phosphate dibasic heptahydrate, $3 \mathrm{~g} / \mathrm{L}$ potassium phosphate monobasic,

$0.5 \mathrm{~g} / \mathrm{L}$ sodium chloride, and $1 \mathrm{~g} / \mathrm{L}$ ammonium chloride.) The microplates were grown in Tecan Infinite $\mathrm{F} 200$ readers with constant shaking at $30 \mathrm{C}$ and with $\mathrm{OD}_{600}$ readings every 15 minutes. For the anaerobic growth curves, we used a plate reader housed in a Coy anaerobic chamber.

Table 1: Oligonucleotide sequences.

\begin{tabular}{|l|l|}
\hline Name & Sequence \\
\hline oAD232 & GGATCCAAACTCGAGTAAGGATCT \\
\hline oAD233 & ATGTATATCTCCTTCTTAAAAGATC \\
\hline oAD793 & AGATCTTTTAAGAAGGAGATATACATATGAGCAAGAGCGCATTCACAT \\
\hline oAD794 & AGATCCTTACTCGAGTTTGGATCCTCAGGCGGCGAAGCTGGCC \\
\hline oAD795 & AGATCTTTTAAGAAGGAGATATACATATGAATATAGTGTTGCCCACATC \\
\hline oAD796 & AGATCCTTACTCGAGTTTGGATCCTCAGGCCGAAAGCTCTCGCC \\
\hline
\end{tabular}

\section{Supplementary Information}




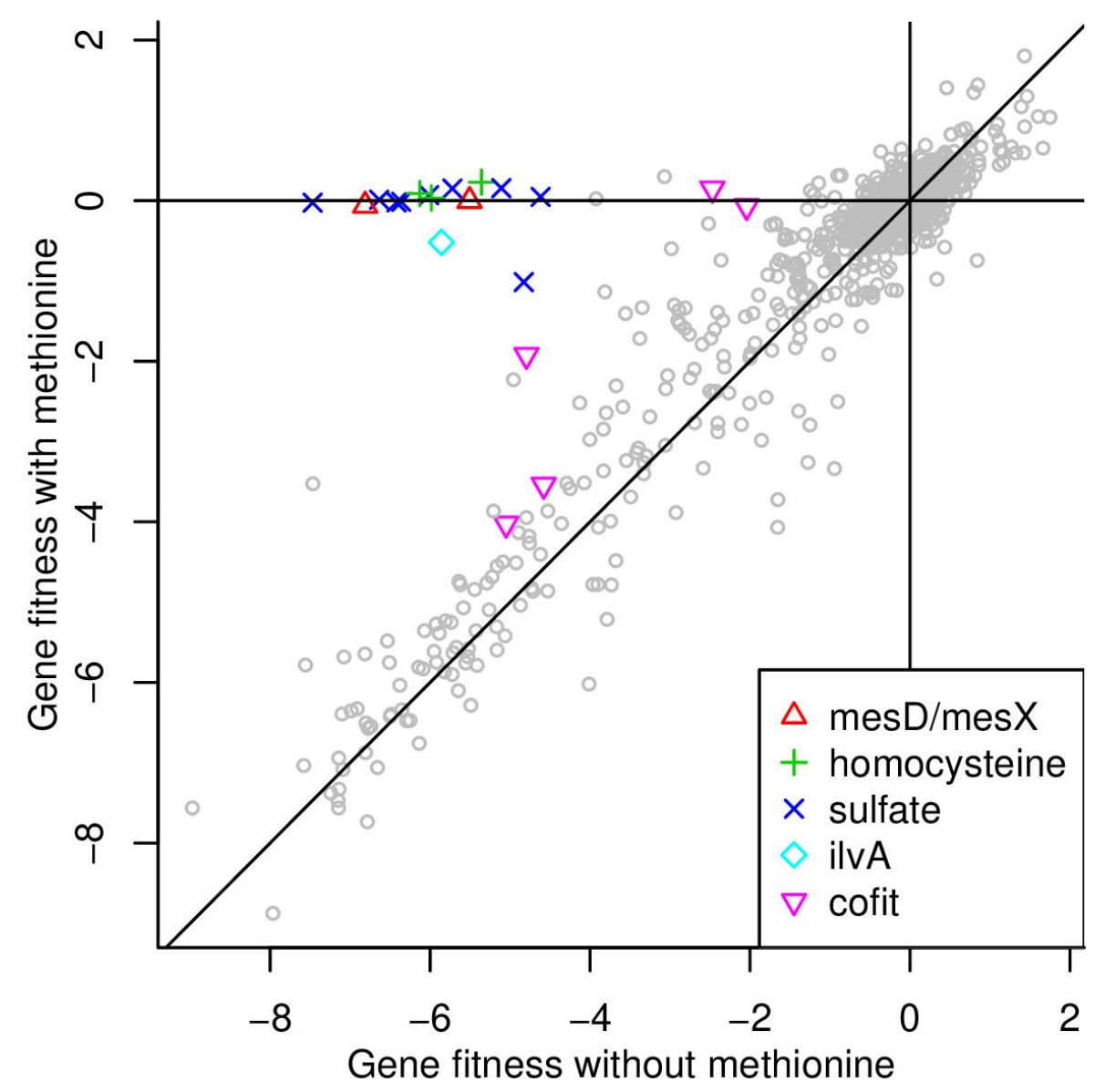

\section{Supplementary Figure S1: Gene fitness from Sphingomonas koreensis growing in} minimal glutamate media with or without methionine. We highlight mesD, mes $X$, genes involved in homocysteine biosynthesis or sulfate assimilation, ilvA, and a few other genes that were cofit with $m e s D$ and were more important for fitness in the absence of methionine. Sulfate is the sulfur source in our defined medium for $S$. koreensis, but sulfate assimilation is not necessary if methionine is available. Mutants in the threonine ammonia-lyase ilvA (Ga0059261_3694), which is the first step in isoleucine biosynthesis, were also rescued by methionine, probably because IlvA's product (2-oxobutanoate) can instead be formed by methionine gamma-lyase (Ga0059261_1458). We also highlight five other genes that were less important for fitness when methionine was added and were cofit with mesD. (We defined cofit as having a Pearson correlation of 0.74 or above, which placed them in the top 20 genes.) These genes included the global regulator $d k s A$ (Ga0059261_0274), genes involved in cell wall biosynthesis (Ga0059261_1360, Ga0059261_2021), a putative sulfur dioxygenase (Ga0059261_2260), and a member of the uncharacterized ApaG family (Ga0059261_3195). The putative dioxygenase has mild phenotypes in many conditions where mes $D$ is very important for fitness, so we do not expect that it is directly involved in methionine biosynthesis by MesD/MesX. ApaG (Ga0059261_3195) is more cofit with sulfate assimilation genes such as Ga0059261_4041 (cysNC, $r=0.87$ ) than with mesD $(r=0.76)$. 


\section{Acknowledgements}

ENIGMA is a Scientific Focus Area Program supported by the U. S. Department of Energy, Office of Science, Office of Biological and Environmental Research, Genomics:GTLFoundational Science through contract DE-AC02-05CH11231 between Lawrence Berkeley National Laboratory and the U. S. Department of Energy.

\section{Bibliography}

Altschul, S. F., T. L. Madden, A. A. Schäffer, J. Zhang, Z. Zhang, W. Miller, and D. J. Lipman. 1997. Gapped BLAST and PSI-BLAST: a new generation of protein database search programs. Nucleic Acids Research 25: 3389-3402. doi:10.1093/nar/25.17.3389.

Baba, T., T. Ara, M. Hasegawa, Y. Takai, Y. Okumura, M. Baba, K. A. Datsenko, M. Tomita, et al. 2006. Construction of Escherichia coli K-12 in-frame, single-gene knockout mutants: the Keio collection. Molecular Systems Biology 2: 2006.0008. doi:10.1038/msb4100050.

Basu, M. K., J. D. Selengut, and D. H. Haft. 2011. ProPhylo: partial phylogenetic profiling to guide protein family construction and assignment of biological process. BMC Bioinformatics 12: 434. doi:10.1186/1471-2105-12-434.

de Berardinis, V., D. Vallenet, V. Castelli, M. Besnard, A. Pinet, C. Cruaud, S. Samair, C. Lechaplais, et al. 2008. A complete collection of single-gene deletion mutants of Acinetobacter baylyi ADP1. Molecular Systems Biology 4: 174. doi:10.1038/msb.2008.10.

Borrel, G., P. S. Adam, and S. Gribaldo. 2016. Methanogenesis and the Wood-Ljungdahl Pathway: An Ancient, Versatile, and Fragile Association. Genome Biology and Evolution 8: 1706-1711. doi:10.1093/gbe/evw114.

Caspi, R., T. Altman, J. M. Dale, K. Dreher, C. A. Fulcher, F. Gilham, P. Kaipa, A. S.

Karthikeyan, et al. 2010. The MetaCyc database of metabolic pathways and enzymes and the BioCyc collection of pathway/genome databases. Nucleic Acids Research 38: D473-9. doi:10.1093/nar/gkp875.

Castresana, J. 2000. Selection of conserved blocks from multiple alignments for their use in phylogenetic analysis. Molecular Biology and Evolution 17: 540-552. doi:10.1093/oxfordjournals.molbev.a026334.

de Crécy-Lagard, V., G. Phillips, L. L. Grochowski, B. El Yacoubi, F. Jenney, M. W. W. Adams, A. G. Murzin, and R. H. White. 2012. Comparative genomics guided discovery of two missing archaeal enzyme families involved in the biosynthesis of the pterin moiety of tetrahydromethanopterin and tetrahydrofolate. ACS Chemical Biology 7: 1807-1816. doi:10.1021/cb300342u.

Crooks, G. E., G. Hon, J. M. Chandonia, and S. E. Brenner. 2004. WebLogo: a sequence logo generator. Genome Research 14: 1188-1190. doi:10.1101/gr.849004.

Dehal, P. S., M. P. Joachimiak, M. N. Price, J. T. Bates, J. K. Baumohl, D. Chivian, G. D. Friedland, K. H. Huang, et al. 2010. MicrobesOnline: an integrated portal for comparative and functional genomics. Nucleic Acids Research 38: D396-400. doi:10.1093/nar/gkp919.

Deobald, D., R. Hanna, S. Shahryari, G. Layer, and L. Adrian. 2020. Identification and 
characterization of a bacterial core methionine synthase. Scientific Reports 10: 2100. doi:10.1038/s41598-020-58873-z.

Durot, M., F. Le Fèvre, V. de Berardinis, A. Kreimeyer, D. Vallenet, C. Combe, S. Smidtas, M. Salanoubat, et al. 2008. Iterative reconstruction of a global metabolic model of Acinetobacter baylyi ADP1 using high-throughput growth phenotype and gene essentiality data. BMC Systems Biology 2: 85. doi:10.1186/1752-0509-2-85.

Edgar, R. C. 2004. MUSCLE: multiple sequence alignment with high accuracy and high throughput. Nucleic Acids Research 32: 1792-1797. doi:10.1093/nar/gkh340.

Ferrer, J.-L., S. Ravanel, M. Robert, and R. Dumas. 2004. Crystal structures of cobalamin-independent methionine synthase complexed with zinc, homocysteine, and methyltetrahydrofolate. The Journal of Biological Chemistry 279: 44235-44238. doi:10.1074/jbc.C400325200.

Finn, R. D., A. Bateman, J. Clements, P. Coggill, R. Y. Eberhardt, S. R. Eddy, A. Heger, K. Hetherington, et al. 2014. Pfam: the protein families database. Nucleic Acids Research 42: D222-30. doi:10.1093/nar/gkt1223.

Fu, H., and W. W. Metcalf. 2015. Genetic basis for metabolism of methylated sulfur compounds in Methanosarcina species. Journal of Bacteriology 197: 1515-1524. doi:10.1128/JB.02605-14.

González, J. C., K. Peariso, J. E. Penner-Hahn, and R. G. Matthews. 1996.

Cobalamin-independent methionine synthase from Escherichia coli: a zinc metalloenzyme. Biochemistry 35: 12228-12234. doi:10.1021/bi9615452.

Goulding, C. W., D. Postigo, and R. G. Matthews. 1997. Cobalamin-dependent methionine synthase is a modular protein with distinct regions for binding homocysteine, methyltetrahydrofolate, cobalamin, and adenosylmethionine. Biochemistry 36: 8082-8091. doi:10.1021/bi9705164.

Haft, D. H., J. D. Selengut, R. A. Richter, D. Harkins, M. K. Basu, and E. Beck. 2013. Tigrfams and genome properties in 2013. Nucleic Acids Research 41: D387-95. doi:10.1093/nar/gks1234.

Harms, U., and R. K. Thauer. 1996. The corrinoid-containing 23-kDa subunit MtrA of the energy-conserving N5-methyltetrahydromethanopterin:coenzyme M methyltransferase complex from Methanobacterium thermoautotrophicum. EPR spectroscopic evidence for a histidine residue as a cobalt ligand of the cobamide. European Journal of Biochemistry / FEBS 241: 149-154. doi:10.1111/j.1432-1033.1996.0149t.x.

Huang, S., G. Romanchuk, K. Pattridge, S. A. Lesley, I. A. Wilson, R. G. Matthews, and M. Ludwig. 2007. Reactivation of methionine synthase from Thermotoga maritima (TM0268) requires the downstream gene product TM0269. Protein Science 16: 1588-1595. doi:10.1110/ps.072936307.

Laskowski, R. A., and M. B. Swindells. 2011. LigPlot+: multiple ligand-protein interaction diagrams for drug discovery. Journal of Chemical Information and Modeling 51: 2778-2786. doi:10.1021/ci200227u.

Lee, T. S., R. A. Krupa, F. Zhang, M. Hajimorad, W. J. Holtz, N. Prasad, S. K. Lee, and J. D. Keasling. 2011. BglBrick vectors and datasheets: A synthetic biology platform for gene expression. Journal of Biological Engineering 5: 12. doi:10.1186/1754-1611-5-12. 
Louca, S., L. W. Parfrey, and M. Doebeli. 2016. Decoupling function and taxonomy in the global ocean microbiome. Science 353: 1272-1277. doi:10.1126/science.aaf4507.

Matthews, R. G., A. E. Smith, Z. S. Zhou, R. E. Taurog, V. Bandarian, J. C. Evans, and M. Ludwig. 2003. Cobalamin-Dependent and Cobalamin-Independent Methionine Synthases: Are There Two Solutions to the Same Chemical Problem? Helvetica chimica acta 86: 3939-3954. doi:10.1002/hlca.200390329.

Mendler, K., H. Chen, D. H. Parks, B. Lobb, L. A. Hug, and A. C. Doxey. 2019. AnnoTree: visualization and exploration of a functionally annotated microbial tree of life. Nucleic Acids Research 47: 4442-4448. doi:10.1093/nar/gkz246.

Menon, A. L., F. L. Poole, A. Cvetkovic, S. A. Trauger, E. Kalisiak, J. W. Scott, S. Shanmukh, J. Praissman, et al. 2009. Novel multiprotein complexes identified in the hyperthermophilic archaeon Pyrococcus furiosus by non-denaturing fractionation of the native proteome. Molecular \& Cellular Proteomics 8: 735-751. doi:10.1074/mcp.M800246-MCP200.

Mwirichia, R., I. Alam, M. Rashid, M. Vinu, W. Ba-Alawi, A. Anthony Kamau, D. Kamanda Ngugi, M. Göker, et al. 2016. Metabolic traits of an uncultured archaeal lineage--MSBL1--from brine pools of the Red Sea. Scientific Reports 6: 19181. doi:10.1038/srep19181.

Pejchal, R., and M. L. Ludwig. 2005. Cobalamin-independent methionine synthase (MetE): a face-to-face double barrel that evolved by gene duplication. PLoS Biology 3: e31. doi:10.1371/journal.pbio.0030031.

Price, M. N., and A. P. Arkin. 2017. PaperBLAST: Text Mining Papers for Information about Homologs. mSystems 2. doi:10.1128/mSystems.00039-17.

Price, M. N., and A. P. Arkin. 2019. Curated BLAST for genomes. mSystems 4. doi:10.1128/mSystems.00072-19.

Price, M. N., P. S. Dehal, and A. P. Arkin. 2010. FastTree 2 - approximately maximum-likelihood trees for large alignments. Plos One 5: e9490. doi:10.1371/journal.pone.0009490.

Price, M. N., K. M. Wetmore, R. J. Waters, M. Callaghan, J. Ray, J. V. Kuehl, R. A. Melnyk, J. S. Lamson, et al. 2016. Deep Annotation of Protein Function across Diverse Bacteria from Mutant Phenotypes. bioRxiv.

Price, M. N., G. M. Zane, J. V. Kuehl, R. A. Melnyk, J. D. Wall, A. M. Deutschbauer, and A. P. Arkin. 2018a. Filling gaps in bacterial amino acid biosynthesis pathways with high-throughput genetics. PLoS Genetics 14: e1007147. doi:10.1371/journal.pgen.1007147.

Price, M. N., K. M. Wetmore, R. J. Waters, M. Callaghan, J. Ray, H. Liu, J. V. Kuehl, R. A. Melnyk, et al. 2018b. Mutant phenotypes for thousands of bacterial genes of unknown function. Nature 557: 503-509. doi:10.1038/s41586-018-0124-0.

Price, M. N., A. M. Deutschbauer, and A. P. Arkin. 2020. Gapmind: automated annotation of amino acid biosynthesis. mSystems 5. doi:10.1128/mSystems.00291-20.

Radle, M. I., D. V. Miller, T. N. Laremore, and S. J. Booker. 2019. Methanogenesis marker protein 10 (Mmp10) from Methanosarcina acetivorans is a radical S-adenosylmethionine methylase that unexpectedly requires cobalamin. The Journal of Biological Chemistry 294: 11712-11725. doi:10.1074/jbc.RA119.007609.

Rozewicki, J., S. Li, K. M. Amada, D. M. Standley, and K. Katoh. 2019. MAFFT-DASH: 
integrated protein sequence and structural alignment. Nucleic Acids Research 47: W5-W10. doi:10.1093/nar/gkz342.

Sah, S., K. Lahry, C. Talwar, S. Singh, and U. Varshney. 2020. Monomeric NADH-Oxidizing Methylenetetrahydrofolate Reductases from Mycobacterium smegmatis Lack Flavin Coenzyme. Journal of Bacteriology 202. doi:10.1128/JB.00709-19.

Sajjaphan, K., N. Shapir, L. P. Wackett, M. Palmer, B. Blackmon, J. Tomkins, and M. J. Sadowsky. 2004. Arthrobacter aurescens TC1 atrazine catabolism genes trzN, atzB, and atzC are linked on a 160-kilobase region and are functional in Escherichia coli. Applied and Environmental Microbiology 70: 4402-4407. doi:10.1128/AEM.70.7.4402-4407.2004.

Schröder, I., and R. K. Thauer. 1999. Methylcobalamin:homocysteine methyltransferase from Methanobacterium thermoautotrophicum. Identification as the metE gene product.

European Journal of Biochemistry / FEBS 263: 789-796. doi:10.1046/j.1432-1327.1999.00559.x.

Shelton, A. N., E. C. Seth, K. C. Mok, A. W. Han, S. N. Jackson, D. R. Haft, and M. E. Taga. 2019. Uneven distribution of cobamide biosynthesis and dependence in bacteria predicted by comparative genomics. The ISME Journal 13: 789-804. doi:10.1038/s41396-018-0304-9.

Sonnhammer, E. L., G. von Heijne, and A. Krogh. 1998. A hidden Markov model for predicting transmembrane helices in protein sequences. Proceedings / ... International Conference on Intelligent Systems for Molecular Biology ; ISMB. International Conference on Intelligent Systems for Molecular Biology 6: 175-182.

Strong, L. C., C. Rosendahl, G. Johnson, M. J. Sadowsky, and L. P. Wackett. 2002. Arthrobacter aurescens TC1 metabolizes diverse s-triazine ring compounds. Applied and Environmental Microbiology 68: 5973-5980. doi:10.1128/aem.68.12.5973-5980.2002.

Taurog, R. E., and R. G. Matthews. 2006. Activation of methyltetrahydrofolate by cobalamin-independent methionine synthase. Biochemistry 45: 5092-5102. doi:10.1021/bi060052m.

UniProt Consortium. 2019. UniProt: a worldwide hub of protein knowledge. Nucleic Acids Research 47: D506-D515. doi:10.1093/nar/gky1049.

Wetmore, K. M., M. N. Price, R. J. Waters, J. S. Lamson, J. He, C. A. Hoover, M. J. Blow, J. Bristow, et al. 2015. Rapid quantification of mutant fitness in diverse bacteria by sequencing randomly bar-coded transposons. mBio 6: e00306-15. doi:10.1128/mBio.00306-15.

Zhuang, W.-Q., S. Yi, M. Bill, V. L. Brisson, X. Feng, Y. Men, M. E. Conrad, Y. J. Tang, et al. 2014. Incomplete Wood-Ljungdahl pathway facilitates one-carbon metabolism in organohalide-respiring Dehalococcoides mccartyi. Proceedings of the National Academy of Sciences of the United States of America 111: 6419-6424. doi:10.1073/pnas.1321542111. 